\title{
Systemic therapy of experimental breast cancer metastases by mutant vesicular stomatitis virus in immune-competent mice
}

\author{
Oliver Ebert, ${ }^{1}$ Sonal Harbaran, ${ }^{1}$ Katsunori Shinozaki, ${ }^{1}$ and Savio LC Woo ${ }^{1}$ \\ ${ }^{1}$ Department of Gene and Cell Medicine, Mount Sinai School of Medicine, New York, New York, USA.
}

In view of the limited success of available treatment modalities for metastatic breast cancer, alternative and complementary strategies need to be developed. Oncolytic vesicular stomatitis virus (VSV) is a promising novel therapeutic agent for the treatment of cancer. The aim of this study was to evaluate the potential of recombinant VSV containing the M51R mutation in the matrix (M) protein gene administered intravenously as an effective and safe therapeutic agent for treating mice with experimental breast cancer metastases. Recombinant VSV(M51R)-LacZ was generated and characterized in vitro on human and murine breast cancer cells. Breast cancer metastases were established in immune-competent Balb/c mice by intravenous injection of syngeneic 4T1 cells. The vector was infused into the tumor-bearing animals via the tail vein, and productive infection of pulmonary breast cancer lesions was assessed by X-gal stainings of frozen lung sections. To evaluate potential systemic toxicity, histology of major organs and serum chemistries were analyzed. To assess effectiveness, buffer- or vector-treated tumor-bearing mice were followed for survival and the results were analyzed by the Kaplan-Meier method and the log-rank test. We found that VSV(M51R)-LacZ efficiently replicated and lysed human breast cancer cells but was partially attenuated in 4T1 cells in vitro. We also demonstrated that its maximum tolerated dose after intravenous infusion in normal Balb/c mice was elevated by at least 100-fold over that of the parental VSV vector containing the wild-type M gene. When VSV(M51R)-LacZ was repeatedly injected intravenously into mice bearing syngeneic 4T1 tumors, the virus was able to infect multiple breast cancer lesions in the lungs without apparent toxicities, which led to significant prolongation of animal survival $(P=.003)$. In conclusion, systemic administration of $M$ mutant VSV is both effective and safe in the treatment of experimental breast cancer metastases in immune-competent mice, suggesting that further development of this approach may have potential for clinical application in patients.

Cancer Gene Therapy (2005) 12, 350-358. doi:10.1038/sj.cgt.7700794

Published online 26 November 2004

Keywords: breast cancer; metastases; oncolytic virus; vesicular stomatitis virus; matrix protein mutant

$I^{\mathrm{n}}$ the United States, breast cancer remains the most common malignancy in women today with approximately 211,000 new cases and 40,000 deaths estimated for 2003. ${ }^{1}$ In some women, breast cancer is a local disease without distant spread. Such early breast cancers are usually diagnosed by screening mammography and are highly curable with local or regional treatment alone. However, most women with primary breast cancer have subclinical metastases, and in a high percentage of those treated with apparently curative surgery (with or without radiotherapy), distant metastases ultimately develop. ${ }^{2}$ The clinical course of metastatic breast cancer is variable. Chemotherapy, hormonal therapy, radiotherapy, and limited surgery are all used in the treatment of women with metastatic breast cancer, although the overwhelming

Received August 4, 2004.

Address correspondence and reprint requests to: $\mathrm{Dr}$ Savio LC Woo, PhD, Department of Gene and Cell Medicine, Mount Sinai School of Medicine, One Gustave L Levy Place, Box 1496, New York, NY 10029-6574,USA. E-mail: savio.woo@mssm.edu

This work was supported in part by NIH Grant R01-CA100830 (to SLCW) and Deutsche Forschungsgemeinschaft Grant EB 234/1-1 (to $O E$ ). majority of these women will die of their disease. ${ }^{2}$ In view of the limited success of available treatment modalities for metastatic breast cancer, alternative and complementary strategies need to be developed.

The use of a molecularly engineered replicationcompetent herpes simplex virus and adenovirus to treat cancer was initially proposed by Martuza et $\mathrm{al}^{3}$ and by McCormick and co-workers ${ }^{4}$ in the 1990 s, respectively, which has been translated into clinical trials recently. ${ }^{5-8}$ In addition, there are a number of viruses with inherent tumor specificities due to attenuated antiviral responses that are manifested by many tumor types, and these are actively being developed as oncolytic agents for cancer treatment. ${ }^{9}$ Vesicular stomatitis virus (VSV) is a nonpathogenic RNA virus that is exquisitely sensitive to the antiviral actions of type 1 interferons (IFNs) in normal but not in cancer cells. ${ }^{10,11}$ This finding has been postulated to be due to the fact that IFN-responsive antiviral pathways are defective in many types of tumors. ${ }^{10,11}$ In addition, defects in translational regulation can cooperate with impaired IFN signaling to facilitate VSV replication in tumor cells. ${ }^{12}$ Compared to other replication-competent oncolytic vectors, VSV is particularly appealing for its rapid replication rate of 
$8-12 \mathrm{~h}$ in tumor cells, ${ }^{13}$ such that significant tumor destruction may have occurred prior to the initiation of potentially neutralizing antiviral immune response in the host. Furthermore, VSV is not endemic to North America, ${ }^{13}$ implying that pre-existing antibodies in patients will not interfere with its infection process after intravascular administration.

Most wild-type (wt) strains of VSV are known to be relatively poor inducers of IFN. ${ }^{14}$ The VSV matrix (M) protein is a virulence factor that is capable of inhibiting host gene expression at the level of transcription, ${ }^{15,16}$ as well as nuclear-cytoplasmic transport of host RNAs and protein. ${ }^{17,18}$ Earlier studies have demonstrated the feasibility of isolating VSV mutants with strong IFN-inducing phenotypes, ${ }^{19}$ with many of these mutants containing point mutations in their $\mathrm{M}$ proteins, including the M51R mutation originally found in the ts082 and T1026R1 mutant viruses. ${ }^{20}$ Recently, Stojdl et $\mathrm{al}^{21}$ demonstrated that VSV M mutants with a single amino-acid (M51R) or two amino-acid (V221F and S226R) substitutions are attenuated in mice because of their potent induction of IFN but retain antitumor activity in vitro and in several animal tumor models, even when applied systemically. ${ }^{21}$ In an alternative approach, Obuchi et $\mathrm{al}^{22}$ have created a recombinant VSV (rVSV) expressing the IFN- $\beta$ gene with enhanced selectivity for tumor cells in tissue culture and in vivo.

In the present study, we tested the hypothesis that an IFN-inducing rVSV(M51R)-LacZ vector containing the M51R mutation would retain specific oncolytic activity in breast cancer cells and, because of its significantly reduced toxicity in normal cells, may be safely administered at higher doses in mice through intravenous infusion. We further hypothesized that experimental breast cancer metastases in the lungs of immune-competent mice may be readily transduced by intravenous infusion of rVSV(M51R)-LacZ vector, which would result in tumor-selective viral replication, oncolysis, and survival advantage of the treated animals.

\section{Materials and methods}

\section{Cell culture}

MCF7, MDA-MB-231, 4T1, and BHK-21 cell lines were obtained from American Type Culture Collection (ATCC; Manassas, VA). MCF7 human breast cancer cells were maintained in MEM-Eagle (Mediatech, Herndon, VA) and supplemented with 10\% FBS (Sigma-Aldrich, St Louis, MO), $2 \mathrm{mM}$ glutamine (Mediatech), $1.5 \mathrm{~g} / 1$ sodium bicarbonate (Mediatech), $0.1 \mathrm{mM}$ nonesssential amino acids (Mediatech), and $1 \mathrm{mM}$ sodium pyruvate (Mediatech). Human breast cancer MDA-MB231 cells were maintained in $10 \%$ FBS-RPMI 1640 (Mediatech). The murine breast cancer cell line 4T1 was maintained in 10\% FBS-RPMI 1640 (Mediatech) and supplemented with $1 \mathrm{mM}$ sodium pyruvate (Mediatech) and $10 \mathrm{mM}$ HEPES (Mediatech). BHK-21 cells used for production and titration of VSV were maintained in $10 \%$
FBS-DMEM (Mediatech). All media used in this study contained $100 \mathrm{U} / \mathrm{ml}$ penicillin-streptomycin (Mediatech).

\section{Constructs and virus generation}

The full-length cDNA VSV clone (plasmid pVSV-XN2 provided by Dr John Rose) was digested with $X b a I$ and $K p n \mathrm{I}$ and the obtained fragment containing the $\mathrm{M}$ protein gene was modified by sire-directed PCR mutagenesis (QuikChange II XL, Stratagene, La Jolla, CA) to generate a single amino-acid substitution from methionine to arginine at position 51 of the $\mathrm{M}$ protein gene. Subsequently, the fragment containing the M51R mutation was ligated into a similar digested full-length cDNA clone of VSV encoding the Escherichia coli $\beta$-galactosidase gene. ${ }^{23}$ Recombinant VSV(M51R)-LacZ virus was generated using the established method of reverse genetics. $^{24,25}$ After successful recovery, vaccinia virus was completely eliminated by double plaque purification, and the titers $(\mathrm{PFU} / \mathrm{ml})$ of viral stocks were determined by standard plaque assays on BHK-21 cells as described previously. $^{26}$

\section{Virus replication assays}

Human and murine breast cancer cells were seeded in sixwell plates at $1 \times 10^{5}$ cells/well overnight and then infected with rVSV-LacZ or rVSV(M51R)-LacZ at a multiplicity of infection (MOI) of 0.01. At indicated time points postinfection, a sample of cell culture supernatant was collected at designated time points after infection and assayed for viral RNA genome by real time RT-PCR using specific primers as described. ${ }^{26}$

\section{In vitro cytotoxicity assays}

Breast cancer cells were seeded in 24-well plates at $1 \times 10^{4}$ cells/well overnight and then infected with rVSVLacZ or rVSV(M51R)-LacZ at an MOI of 0.01. Cell viability was measured at the indicated time points after infection using the 3-(4,5-dimethylthiazol-2-yl)-2,5-diphenyltetrazolium bromide (MTT) assay (Cell Proliferation Kit I, Roche, Indianapolis, IN). All cell viability results are expressed as percentage of viable cells compared to mock-infected control at each time point.

\section{Animal studies}

Female Balb/c mice (6-7 weeks old) were purchased from Charles River Laboratories Inc. (Wilmington, MA) and housed in a specific pathogen-free environment under standard conditions. All procedures involving animals were approved by and performed according to guidelines of the Institutional Animal Care and Use Committee of the Mount Sinai School of Medicine. In order to establish experimental breast cancer metastases, mice were injected with $3.3 \times 10^{4}$ syngeneic murine $4 \mathrm{~T} 1$ cells in $100 \mu \mathrm{l}$ of PBS via the tail vein. At 12 days after tumor cell infusion, animals were infused intravenously with rVSV-LacZ or rVSV(M51R)-LacZ vector in $100 \mu \mathrm{l}$ of PBS or an equivalent volume of PBS buffer. To evaluate the kinetics of viral replication within the lung tumor lesions, sets of 
animals were killed at various time points after intravenous infusion of VSV. Tissues samples were obtained and subjected to plaque assays to determine the viral yield as described. ${ }^{26}$ In a separate experiment, groups of tumorbearing animals infused with either rVSV(M51R)-LacZ or PBS buffer control were followed for survival, which was checked daily in all animals.

\section{Histology and X-gal staining}

At indicated time points after vector infusion, animals were killed and various major organs (brain, liver, heart, spleen, kidneys, lungs) were fixed in 4\% paraformaldehyde overnight and then paraffin-embedded. Sections $(5 \mu \mathrm{m})$ were subjected to hematoxylin and eosin $(\mathrm{H} \& \mathrm{E})$ staining for histological analysis. In addition, frozen tumor-bearing lung sections were stained in 5-bromo4-chloro-3-indolyl- $\beta$-D-galactopyranoside (X-gal) staining solution to evaluate intratumoral virus spread. ${ }^{23}$ X-gal-stained frozen sections were counterstained with hematoxylin.

\section{Assessment of serum chemistries}

Blood samples were collected from the retro-orbital vein before and at days 1, 2, 3, and 4 after virus injection, and the levels of serum alanine aminotransferase (ALT) and creatinine were determined at the Chemistry Laboratory at Mount Sinai School of Medicine.

\section{Statistical analyses}

Survival curves of animals treated with VSV vector or buffer were plotted according to the Kaplan-Meier method. Statistical significance in different treatment groups was compared using the log-rank test. Results and graphs were obtained using the GraphPad Prism 3.0 program (GraphPad Software, San Diego, CA).

\section{Results}

$M$ mutant $V S V$ retains its replication potential and cytotoxicity in human breast cancer cells in vitro

To compare replication and cytotoxicity of $\mathrm{M}$ mutant versus wtVSV in breast cancer cells in vitro, the murine (4T1) and human (MDA-MB-231 and MCF7) breast cancer cell lines were examined in virus infection assays (Fig 1). Cells were infected with rVSV-LacZ or rVSV(M51R)-LacZ at a low MOI of 0.01, and the supernatants were harvested at various time points postinfection. To test the correlation between standard plaque assays and our recently established real-time RTPCR,${ }^{26}$ we first analyzed supernatants from VSV-infected BHK-21 cells $(n=5)$ simultaneously using both assays. We found strong correlation with a Pearson's correlation coefficient of 0.99 , which was highly significant $(P=.0046)$. Total RNAs from the breast cancer cell culture supernatants were prepared, and the RNA samples were analyzed for the presence and concentrations of a
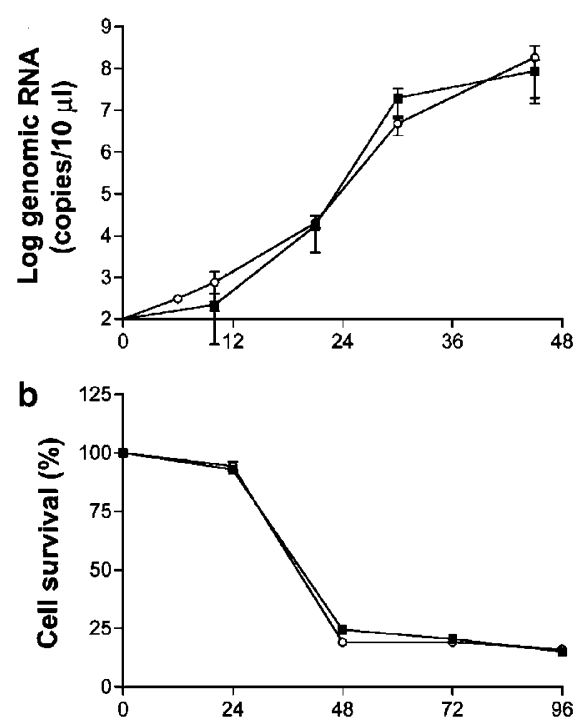

MCF7
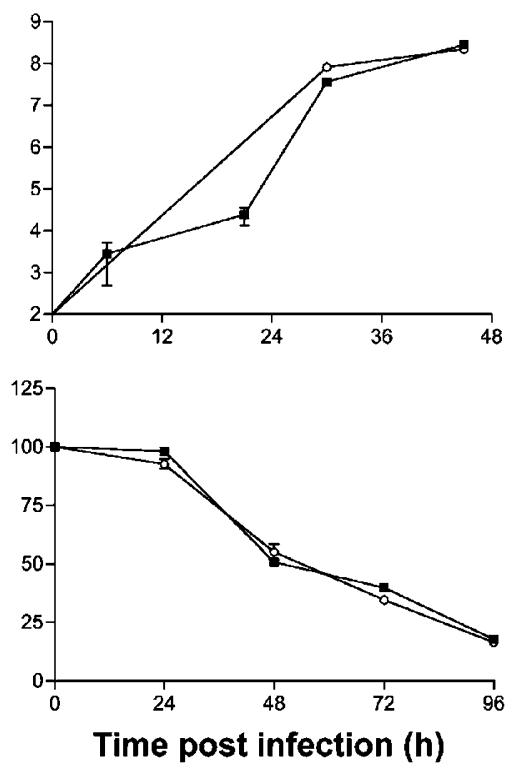

4T1
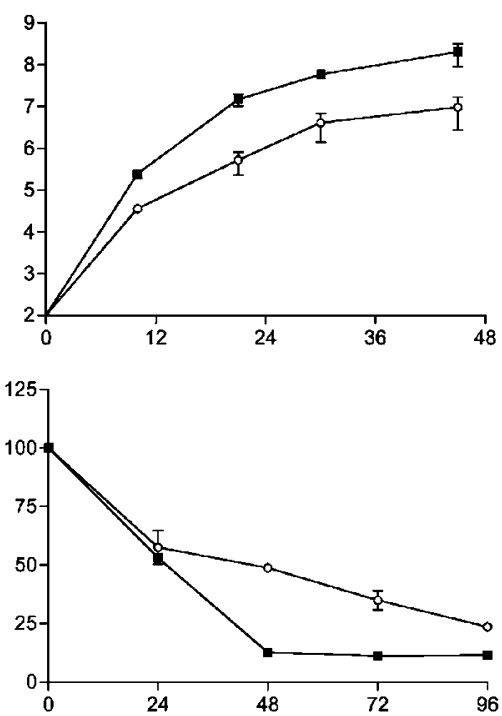

Figure 1 in vitro effects of rVSV(M51R)-LacZ (open circle) and rVSV-LacZ (black square) on murine and human breast cancer cells. Human (MDA-MB-231, MCF7) and murine (4T1) breast cancer cells were infected with the viruses at an MOI of 0.01, and at the indicated time points samples of supernatant were assayed by (a) quantitative RT-PCR for the presence and concentrations of viral genomic RNA and (b) for cell viability by an MTT assay. The $\mathrm{OD}_{570}$ values of mock-infected cells were set as $100 \%$ at each time point. Data are expressed as means \pm standard error of triplicate experiments. 
genomic VSV RNA by real-time RT-PCR. The results showed that M mutant VSV possessed the same capacity of replicating its RNA genome in human breast cancer cells compared to wtVSV and similarly reached plateau within 24-48 hours of inoculation (Fig 1a). On the other hand, replication of $M$ mutant VSV in murine 4T1 cells was substantially attenuated, indicating that 4T1 cells may retain partial sensitivity to the antiviral activity of IFN.

In addition, the cytotoxic effects of both viruses on the human and murine breast cancer cells were quantified by MTT assays (Fig 1b). The kinetic profiles of cell killing caused by either virus were very similar in the human breast cancer cell lines, and most cells were eradicated within 48-96 hours postinfection. As expected, the degree of M mutant VSV killing of murine 4T1 cells was not as rapid as seen in the wtVSV-infected cells, indicating that 4T1 cells were more resistant to M mutant VSV-mediated cell killing. However, most 4T1 cells were eradicated by M mutant VSV within 96 hours postinfection, which was comparable with the human breast cancer cell line MCF7. The results showed that $\mathrm{M}$ mutant VSV retained its replication potential and cytotoxicity in human breast cancer cells in vitro but was partially attenuated in the murine 4T1 breast cancer cell line.

\section{The MTD of M mutant VSV administered intravenously} in mice is significantly elevated

To evaluate the toxicity profiles and maximum tolerated doses (MTD) of M mutant and wtVSV in vivo, normal $\mathrm{Balb} / \mathrm{c}$ mice were randomized to receive buffer or escalating doses of the rVSV(M51R)-LacZ or rVSV-LacZ vectors administered intravenously in $100 \mu \mathrm{l}$ in half-log increments that ranged from $1.3 \times 10^{6}$ to $1.3 \times 10^{8} \mathrm{PFU}$ (maximum concentration that can currently be produced) (Fig 2). The animals were closely monitored for weight loss, dehydration, piloerection, and hind limb paralysis or other neurotoxic signs and killed if necessary as per good laboratory standards. There were 10 animals per vector dose and the results showed that the MTD of rVSV(M51R)-LacZ was elevated by $>100$-fold over that of rVSV-LacZ containing the wt M gene, indicating that rVSV(M51R)-LacZ is a much safer vector compared to its parental counterpart.

Mice treated with $\mathrm{rVSV}(\mathrm{M} 51 \mathrm{R})-\mathrm{LacZ}$ at $4.0 \times 10^{7} \mathrm{PFU}$ doses or higher experienced significant weight losses of up to $15 \%$ body weight but rapidly recovered after day 4 post virus infusion. Stojdl et $\mathrm{al}^{21}$ have demonstrated that $\mathrm{M}$ mutant, in contrast to wtVSV, induces strongly elevated antiviral responses and inflammatory cytokine expression in the infected cells. We therefore suspect that the production of pro-inflammatory cytokines by $4 \mathrm{~T} 1$ cells infected by the $\mathrm{M}$ mutant virus were likely to be responsible for the observed weight loss in the treated animals. Animals treated with repeated systemic injections of the M mutant VSV vector at $1.3 \times 10^{8} \mathrm{PFU} /$ dose experienced body weight loss of up to $10 \%$ following the initial treatment at day 2 , and all subsequent doses failed to elicit any signs of further body weight loss.
Access to pulmonary breast cancer lesions and tumor-selective replication of M mutant VSV after intravenous infusion

To develop a treatment strategy for patients with metastatic breast cancer, we tested the hypothesis that experimental breast cancer metastases in immune-competent mice can be readily accessed by rVSV(M51R)-LacZ via intravenous administration, which will lead to tumorselective viral replication and spread, oncolysis, and survival advantage of the treated animals. To this end, we have established an immune-competent $\mathrm{Balb} / \mathrm{c}$ breast cancer mouse model as previously described. ${ }^{27}$ Briefly, $3.3 \times 10^{4}$ syngeneic $4 \mathrm{~T} 1$ murine breast cancer cells in $100 \mu \mathrm{l}$ were infused into the tail vein. After 12 days, multiple pulmonary lesions (3-5 tumor nodules per lung section, up to $1 \mathrm{~mm}$ in diameter) could be observed histologically in $90-100 \%$ of animals, and $\operatorname{rVSV}(\mathrm{M} 51 \mathrm{R})$ LacZ vector $\left(1.3 \times 10^{8} \mathrm{PFU}\right)$ in $100 \mu \mathrm{l}$ was then infused intravenously into the animals.

To evaluate whether rVSV(M51R)-LacZ administered intravenously could gain access to and selectively replicate in pulmonary breast cancer lesions, we killed three tumorbearing animals at 30 minutes and 1,2, 3, 4 days after a single infusion with recombinant VSV vector. The lungs were explanted, and frozen lung sections containing normal lung and tumor tissues were obtained and subjected to X-gal staining (Fig 3a). No $\beta$-galactosidase (LacZ) expression was evident at 30 minutes (data not shown), while LacZ expression was restricted to the tumors in virus-injected animals at day 1 post virus infusion. In addition, small necrotic areas were apparent within the tumor lesions at day 1 after virus infusion (Fig $3 b)$. Therefore, our results indicate that $\operatorname{rVSV}(M 51 \mathrm{R})$ LacZ readily gained access to, and productively transduced, multiple pulmonary breast cancer lesions after intravenous administration.

To determine quantitatively the extents of viral replication in the tumors, whole lung tissues were harvested at each time point, mechanically homogenized, centrifuged to remove cellular debris, and the supernatants used to perform plaque assays on BHK-21 cells (Fig 3c). The results indicated that there was a 30-fold increase in infectious virus yield in the lung lesions from 30 minutes to day 1 post vector infusion. Virus yield decreased rapidly after day 1 and became undetectable at day 3 , indicating that intratumoral virus replication was not sustained over time in the immune-competent host.

\section{Absence of organ toxicity after intravenous infusion of $M$ mutant VSV in tumor-bearing mice}

To assess potential organ toxicity after intravenous administration of $\mathrm{rVSV}(\mathrm{M} 51 \mathrm{R})-\mathrm{LacZ}$ vector at $1.3 \times 10^{8} \mathrm{PFU}$ in tumor-bearing Balb/c mice, the kinetic profiles of serum ALT and creatinine levels as measures of liver and kidney damage, respectively, were determined in the same experimental animal groups above (Fig 4). Mild elevation of serum ALT was seen at day 1 after intravenous administration of VSV(M51R)-LacZ, but 


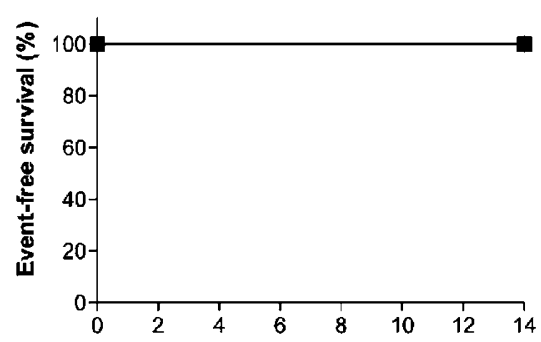

b
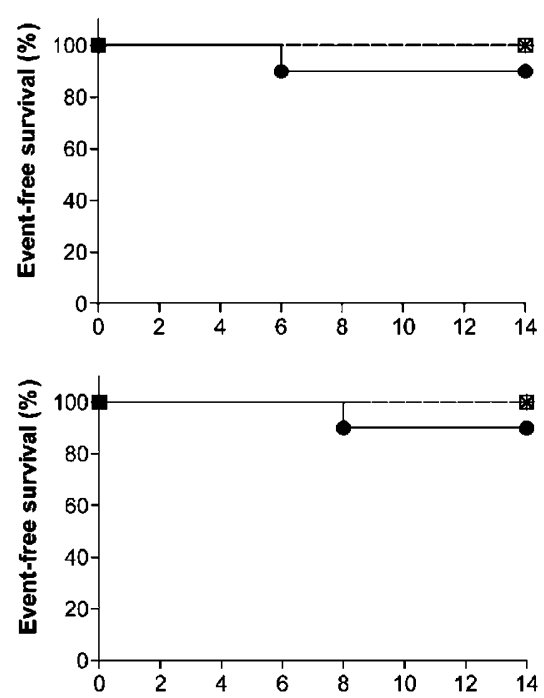

d

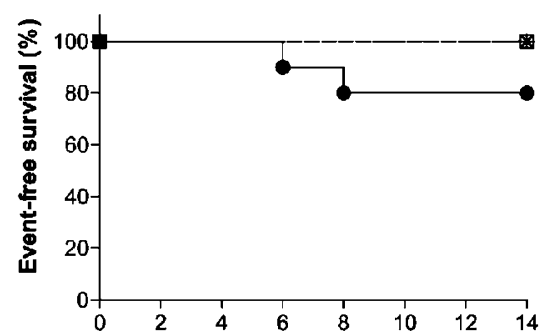

e

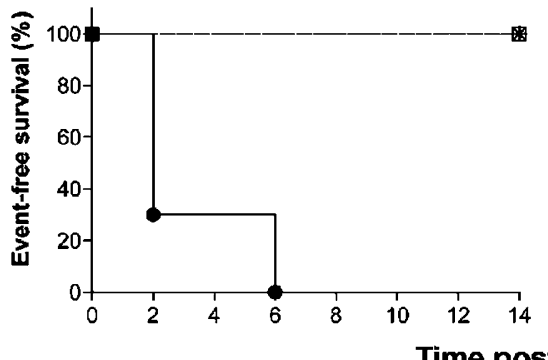

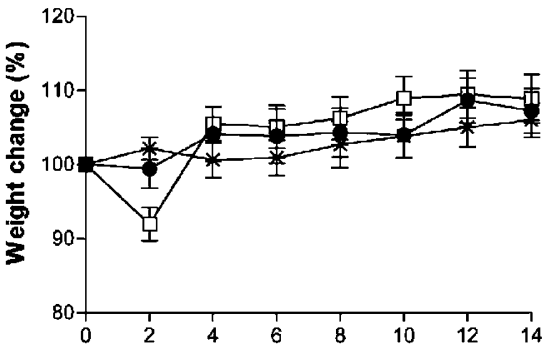
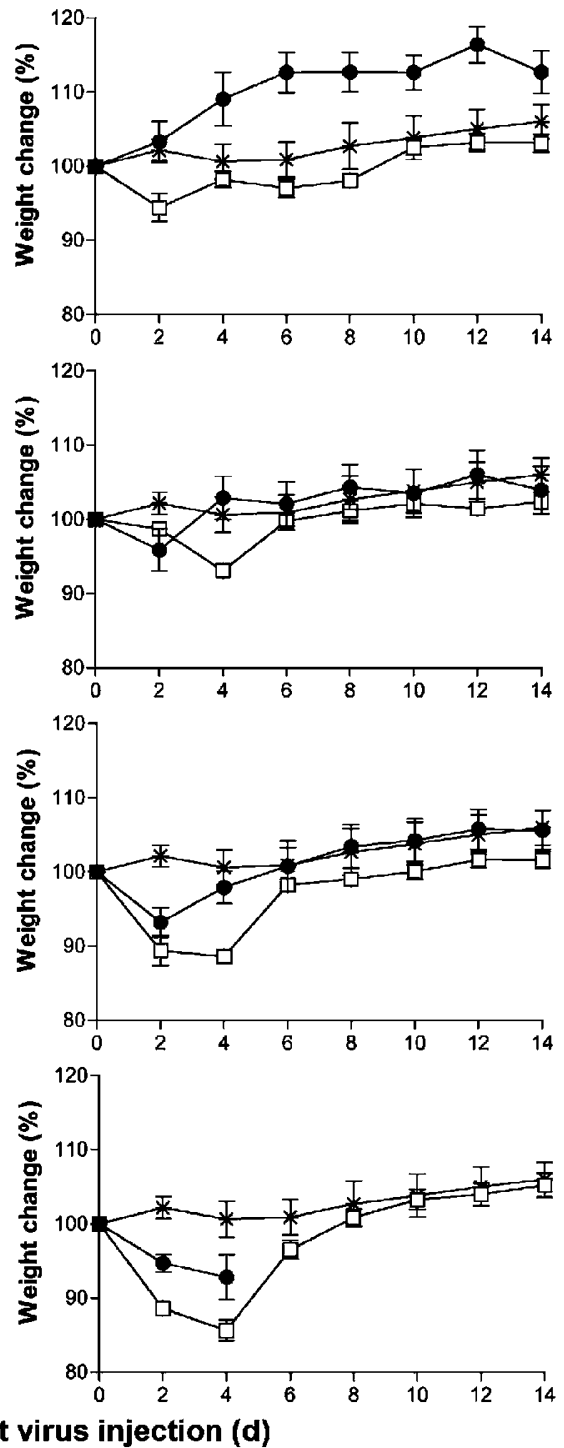

Figure 2 Determination of the MTD after intravenous infusion of rVSV(M51R)-LacZ or rVSV-LacZ. Normal Balb/c mice ( $n=10 /$ dose level cohort) were randomly assigned to a single infusion at day 0 with PBS (star), rVSV(M51R)-LacZ (open square), or parental rVSV-LacZ (black circle) at the following doses: (a) $1.3 \times 10^{6} \mathrm{PFU}$, (b) $4.0 \times 10^{6} \mathrm{PFU}$, (c) $1.3 \times 10^{7} \mathrm{PFU}$, (d) $4.0 \times 10^{7} \mathrm{PFU}$, (e) $1.3 \times 10^{8} \mathrm{PFU}$. Animals were monitored over a period of 14 days for event-free survival (left panel) and for weight changes (right panel).

the levels rapidly returned to baseline at day 2 . There were no elevations of creatinine at all time points, indicating a lack of nephrotoxicity. Additionally, the histology of major organs including the liver was completely normal (data not shown). The results confirmed the notion that there was no remarkable toxicity associated with intravenous infusion of rVSV(M51R)-LacZ at the selected dose.

\section{In vivo antitumor efficacy}

To assess the potential of $\operatorname{rVSV}(\mathrm{M} 51 \mathrm{R})-\mathrm{LacZ}$ as a therapeutic agent for metastatic breast cancer, 4T1 tumor-bearing mice (12 days after tumor cell inoculation) were randomly assigned to infusion with $\operatorname{rVSV}(\mathrm{M} 51 \mathrm{R})$ LacZ at $1.3 \times 10^{8} \mathrm{PFU} /$ dose or PBS control via the tail 

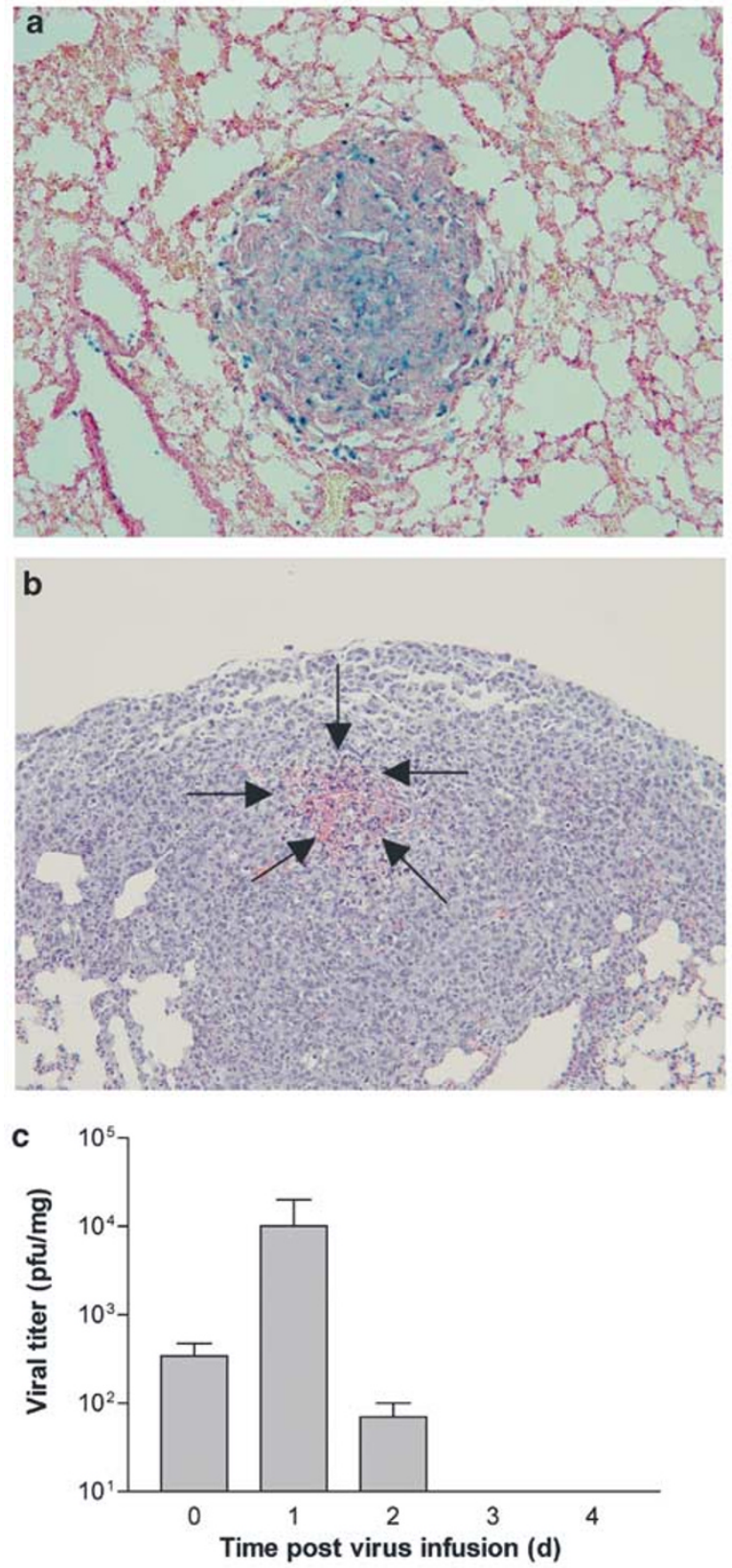

Figure 3 Efficient transduction and tumor-selective replication of rVSV(M51R)-LacZ (1.3 × $\left.10^{8} \mathrm{PFU}\right)$ after single intravenous administration in mice bearing pulmonary breast cancer metastases. (a) Sets of animals $(n=3)$ infused with virus were killed at day 1 after treatment and frozen lung sections were stained with X-gal for $\beta$-gal expression. A representative picture showing a tumor nodule with surrounding lung tissue is shown (magnification, $\times 40$ ). (b) $\mathrm{H} \& \mathrm{E}$ staining of a representative section of a pulmonary 4T1 nodule with necrotic area (arrows) obtained at day 1 after treatment (magnification, $\times 10)$. (c) Viral titers (PFU/mg) in lung tissue samples obtained from tumor-bearing animals at 30 minutes (time point 0 ) and days 1 , 2,3 , and 4 post virus infusion (mean \pm standard error; $n=3$ /time point). Tissue samples were obtained for infectious virus extraction, and the samples were analyzed by plaque assay. vein once every other day for 2 weeks (six applications total), and survival was followed (Fig 5). Buffer-treated mice started to die of tumor progression in 4 more days and all of them expired at 8 days. The VSV-treated mice survived for up to 11 days post vector injection, and the differential survival rates were statistically significant by log-rank test analysis $(P=.003)$. This experiment indicated the remarkable ability of rVSV(M51R)-LacZ to produce a statistically significant prolongation of animal survival in this highly tumorigenic and nonimmunogenic model of breast cancer metastases.

\section{Discussion}

In this communication, we have explored the potential of an IFN-inducing VSV mutant as an effective and safe oncolytic agent for treatment of breast cancer metastases via intravenous administration in an immune-competent mouse model. We have constructed an $\operatorname{rVSV}(\mathrm{M} 51 \mathrm{R})$ LacZ vector containing the M51R mutation in the $\mathrm{M}$ protein gene and expressing the E. coli $\beta$-galacatosidase gene, and showed that the virus retained its oncolytic potential against human breast cancer cell lines in vitro. The general applicability of $\mathrm{M}$ mutant VSV strains as effective agents to kill multiple tumor types in vitro was also demonstrated by Bell's group showing that these viruses are highly lytic in most of the NCI 60 panel of human cancer cell lines. ${ }^{21}$ Their studies further demonstrated that infection with M mutant VSV can establish an antiviral state in the recipient animals that protects against toxicities associated with infection of healthy cells. ${ }^{21}$ We have confirmed their observation in our immune-competent mouse model by demonstrating that the MTD of M mutant VSV after intravenous infusion in normal Balb/c mice was elevated by at least 100 -fold over that of rVSV-LacZ containing the wt $\mathrm{M}$ gene. Thus, the $\mathrm{M}$ mutant VSV is a much safer oncolytic virus for cancer treatment as compared to the wtVSV.

To evaluate the effect of systemic rVSV(M51R)-LacZ treatment on efficacy against breast cancer metastases in vivo, we have chosen the experimental murine 4T1 lung tumor model, which is known to be highly tumorigenic, invasive, and nonimmunogenic. ${ }^{27,28}$ The previously reported study on a similar syngeneic lung tumor model was established with murine CT26 colorectal carcinoma cells, ${ }^{21}$ which are known to be partially immunogenic. Therefore, it is possible that the much more dramatic tumor response in the CT26 lung tumor model after treatment with a very similar VSV mutant may be attributable to antitumor immune responses that contributed to the exceptional treatment outcome. In order to evaluate the potency of the $\mathrm{M}$ mutant virus as an oncolytic agent to treat nonimmunogenic tumors, we have chosen the nonimmunogenic 4T1 breast carcinoma model in immune-competent and syngeneic mice. The results illustrated that the $M$ mutant was only partially effective in this tumor model, and a variety of nonimmunogenic tumors types will need to be further tested in appropriate animal models in future studies. 


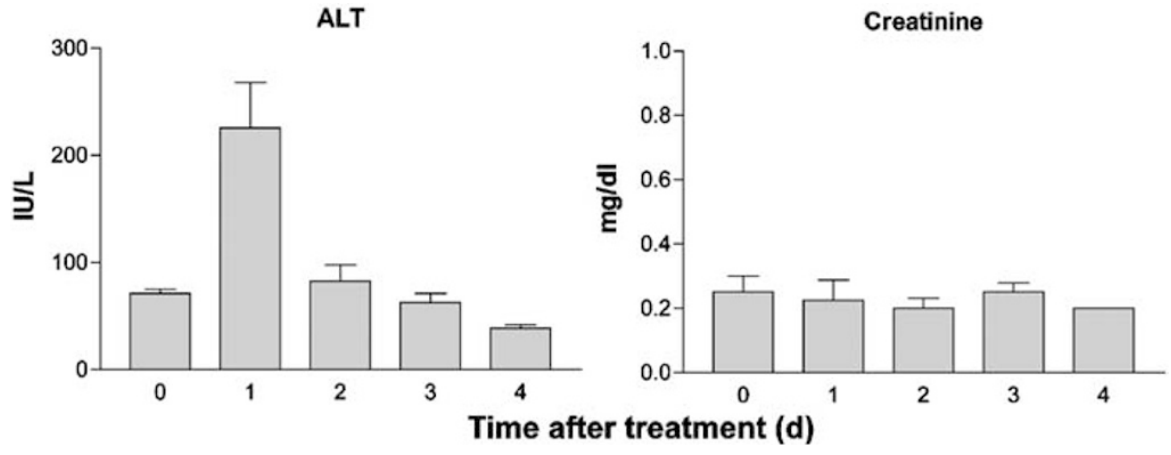

Figure 4 Serum ALT and creatinine levels in tumor-bearing animals (mean \pm standard error; $n=3$ /time point) after a single intravenous infusion of rVSV(M51R)-LacZ at $1.3 \times 10^{8} \mathrm{PFU}$. Blood samples were collected from the same sets of animals as in Figure 3 at indicated time points (time point 0 ; before virus injection) and serum chemistry was performed.

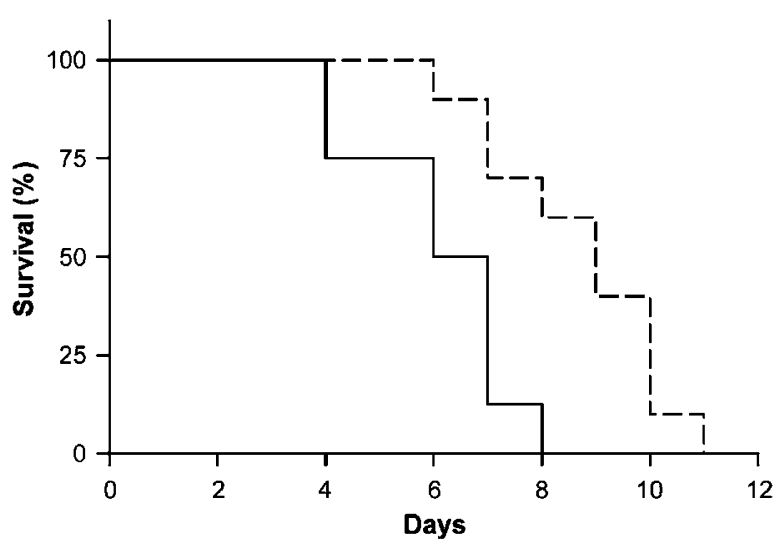

Figure 5 Kaplan-Meier survival curve of mice with 4T1 breast cancer metastases after repeated intravenous infusion of rVSV(M51R)-LacZ versus PBS. Lung tumors were established by injecting $3.3 \times 10^{4}$ murine breast cancer $4 \mathrm{~T} 1$ cells into the tail vein of syngeneic Balb/c mice. On day 12, mice were treated with PBS control $\left(n=8\right.$; solid line) or $1.3 \times 10^{8} \mathrm{PFU}$ VSV vector $(n=10$; dashed line) once every other day for 2 weeks by intravenous injections. The survival advantage for VSV-administered animals was statistically significant compared with PBS control animals $(P=.003$, log-rank test).

In this study, we provide conclusive evidence that $\mathrm{M}$ mutant VSV can gain access to breast cancer metastases in the lungs after intravenous administration and replicate selectively within the tumors. Compared with our previously published results on wtVSV treatment of hepatocellular carcinoma in rats and colorectal carcinoma in mice showing more than 1000-fold increases in infectious virus yields in the tumor lesions, ${ }^{23,26,29}$ intratumoral replication of $\mathrm{M}$ mutant VSV in $4 \mathrm{~T} 1$ breast cancer in mice of only 30 -fold is relatively modest. It is possible that the VSV mutant, by virtue of its defect in shutting down host gene expression, induces robust innate antiviral immune responses that in turn severely attenuate intratumoral virus replication. The average wet lung weight of the 4T1 tumor-bearing animals was approximately $200 \mathrm{mg}$ at the time of killing 1 day after virus treatment (data not shown). Therefore, the absolute recovered titer was $2 \times 10^{6} \mathrm{PFU}$, which was almost 2-log below the input virus dose of $1.3 \times 10^{8} \mathrm{PFU}$. However, compared with the amount of virus recovered from the lung directly after virus infusion, there was a 30 -fold increase in total virus yield (Fig 3c), indicating that the virus has replicated to some degree in the tumor nodules. Virus titer in 4T1 tumors peaked at day 1 and became undetectable after day 2. This correlates with the emergence of T-cell-independent neutralizing $\operatorname{IgM}$ antibodies that occur very early after VSV infection, by day 3 or $4 .{ }^{30,31}$ Due to the rapid replication rate of the virus in tumor cells, however, substantial oncolytic effects have manifested before the onset of neutralizing antiviral immune responses, which led to significant prolongation of animal survival.

The same results also indicated, however, that the mutant VSV-mediated oncolysis was only partially effective and all treated animals eventually succumbed to tumor relapse, indicating the need for an improved viral vector. The development of a "reverse genetics" system for negative-sense RNA viruses has made it possible to engineer the genome of VSV for the construction of novel gene therapy vectors. ${ }^{24,25}$ Recombinant VSVs expressing the herpes simplex virus thymidine kinase or the cytosine deaminase suicide genes have been reported by Barber's group. ${ }^{32,33}$ Their results suggest that the use of the prodrug-suicide gene strategy may add synergy to the effectiveness of oncolytic VSV in cancer treatment. An alternative strategy of cytotoxic gene therapy was recently introduced by Bateman et al, ${ }^{34}$ demonstrating that plasmid-mediated expression of viral fusogenic membrane glycoprotein efficiently killed tumor cells through induction of fusion to form large multinucleated syncytia. In vitro, this "bystander effect" was reported to be at least 10 -fold greater than that observed with various suicide gene/prodrug systems. ${ }^{34}$ In this regard, we reported the construction of a novel recombinant VSV expressing a mutant fusion gene from Newcastle disease virus capable of inducing syncytia formation between tumor cells through membrane fusion at neutral $\mathrm{pH}$, which led to enhanced viral transmission and oncolysis of hepatocellular carcinoma in the livers of rats. ${ }^{35}$ This and other transgenes can be integrated into $\mathrm{M}$ 
mutant VSVs to increase their potential for the treatment of breast cancer metastases further.

In summary, metastatic breast cancer remains a significant clinical problem with limited therapeutic options. Here, we show that intravenous administration of an IFN-inducing VSV vector is both effective and safe in the treatment of experimental breast cancer metastases in immune-competent mice, suggesting that further development of this approach may have potential for clinical application in patients.

\section{Acknowledgments}

We thank Dr John Rose of Yale University for providing pVSV-XN2 plasmid DNA, Dr Tian-Gui Huang for discussions, Dr John Fallon for consultation on histological analyses of tissue samples, and $\mathrm{Ms}$ Jing $\mathrm{Xu}$ for excellent technical assistance.

\section{References}

1. Jemal A, Murray T, Samuels A, et al. Cancer statistics, 2003. CA Cancer J Clin. 2003;53:5-26.

2. Hortobagyi GN. Treatment of breast cancer. $N$ Engl J Med. 1998:339:974-984.

3. Martuza RL, Malick A, Markert JM, et al. Experimental therapy of human glioma by means of a genetically engineered virus mutant. Science. 1991;252:854-856.

4. Bischoff JR, Kirn DH, Williams A, et al. An adenovirus mutant that replicates selectively in p53-deficient human tumor cells. Science. 1996;274:373-376.

5. Khuri FR, Nemunaitis J, Ganly I, et al. A controlled trial of intratumoral ONYX-015, a selectively-replicating adenovirus, in combination with cisplatin and 5-fluorouracil in patients with recurrent head and neck cancer. Nat Med. 2000;6:879-885.

6. Nemunaitis J, Ganly I, Khuri F, et al. Selective replication and oncolysis in p53 mutant tumors with ONYX-015, an E1B-55kD gene-deleted adenovirus, in patients with advanced head and neck cancer: a phase II trial. Cancer Res. 2000;60:6359-6366.

7. Ganly I, Kirn D, Eckhardt G, et al. A phase I study of Onyx-015, an E1B attenuated adenovirus, administered intratumorally to patients with recurrent head and neck cancer. Clin Cancer Res. 2000;6:798-806.

8. Markert JM, Medlock MD, Rabkin SD, et al. Conditionally replicating herpes simplex virus mutant, G207 for the treatment of malignant glioma: results of a phase I trial. Gene Therapy. 2000;7:867-874.

9. Kirn D, Martuza RL, Zwiebel J. Replication-selective virotherapy for cancer: biological principles, risk management and future directions. Nat Med. 2001;7:781-787.

10. Stojdl DF, Lichty B, Knowles S, et al. Exploiting tumorspecific defects in the interferon pathway with a previously unknown oncolytic virus. Nat Med. 2000;6:821-825.

11. Balachandran S, Barber GN. Vesicular stomatitis virus (VSV) therapy of tumors. IUBMB Life. 2000;50:135-138.

12. Balachandran S, Barber GN. Defective translational control facilitates vesicular stomatitis virus oncolysis. Cancer Cell. 2004;5:51-65.
13. Rose JK, Whitt MA. Rhabdoviridae: the viruses and their replication. In: Knipe DM, Howley PM, eds. Fields Virology. Philadelphia: Lippincott Williams \& Wilkins; 2001: 1221-1242.

14. Marcus PI, Rodriguez LL, Sekellick MJ. Interferon induction as a quasispecies marker of vesicular stomatitis virus populations. J Virol. 1998;72:542-549.

15. Ferran MC, Lucas-Lenard JM. The vesicular stomatitis virus matrix protein inhibits transcription from the human beta interferon promoter. $J$ Virol. 1997;71:371-377.

16. Ahmed M, McKenzie MO, Puckett S, et al. Ability of the matrix protein of vesicular stomatitis virus to suppress beta interferon gene expression is genetically correlated with the inhibition of host RNA and protein synthesis. $J$ Virol. 2003;77:4646-4657.

17. Petersen JM, Her LS, Varvel V, et al. The matrix protein of vesicular stomatitis virus inhibits nucleocytoplasmic transport when it is in the nucleus and associated with nuclear pore complexes. Mol Cell Biol. 2000;20:85908601

18. von Kobbe C, van Deursen JM, Rodrigues JP, et al Vesicular stomatitis virus matrix protein inhibits host cell gene expression by targeting the nucleoporin Nup98. Mol Cell. 2000;6:1243-1252.

19. Francoeur AM, Poliquin L, Stanners CP. The isolation of interferon-inducing mutants of vesicular stomatitis virus with altered viral $\mathrm{P}$ function for the inhibition of total protein synthesis. Virology. 1987;160:236-245.

20. Desforges M, Charron J, Berard S, et al. Different host-cell shutoff strategies related to the matrix protein lead to persistence of vesicular stomatitis virus mutants on fibroblast cells. Virus Res. 2001;76:87-102.

21. Stojdl DF, Lichty BD, tenOever BR, et al. VSV strains with defects in their ability to shutdown innate immunity are potent systemic anti-cancer agents. Cancer Cell. 2003;4: 263-275.

22. Obuchi M, Fernandez M, Barber GN. Development of recombinant vesicular stomatitis viruses that exploit defects in host defense to augment specific oncolytic activity. J Virol. 2003;77:8843-8856.

23. Shinozaki K, Ebert O, Kournioti C, et al. Oncolysis of multifocal hepatocellular carcinoma in the rat liver by hepatic artery infusion of vesicular stomatitis virus. Mol Ther. 2004;9:368-376.

24. Lawson ND, Stillman EA, Whitt MA, et al. Recombinant vesicular stomatitis viruses from DNA. Proc Natl Acad Sci USA. 1995;92:4477-4481.

25. Whelan SP, Ball LA, Barr JN, et al. Efficient recovery of infectious vesicular stomatitis virus entirely from cDNA clones. Proc Natl Acad Sci USA. 1995;92:8388-8392.

26. Ebert O, Shinozaki K, Huang TG, et al. Oncolytic vesicular stomatitis virus for treatment of orthotopic hepatocellular carcinoma in immune-competent rats. Cancer Res. 2003;63:3605-3611.

27. Morecki S, Yacovlev E, Diab A, et al. Allogeneic cell therapy for a murine mammary carcinoma. Cancer Res. 1998;58:3891-3895.

28. Pulaski BA, Ostrand-Rosenberg S. Reduction of established spontaneous mammary carcinoma metastases following immunotherapy with major histocompatibility complex class II and B7.1 cell-based tumor vaccines. Cancer Res. 1998;58:1486-1493.

29. Huang TG, Ebert O, Shinozaki K, et al. Oncolysis of hepatic metastasis of colorectal cancer by recombinant 
vesicular stomatitis virus in immune-competent mice. Mol Ther. 2003;8:434-440.

30. Charan S, Zinkernagel RM. Antibody mediated suppression of secondary $\operatorname{IgM}$ response in nude mice against vesicular stomatitis virus. J Immunol. 1986;136:3057-3061.

31. Kundig TM, Schorle H, Bachmann MF, et al. Immune responses in interleukin-2-deficient mice. Science. 1993;262: 1059-1061.

32. Fernandez M, Porosnicu M, Markovic D, et al. Genetically engineered vesicular stomatitis virus in gene therapy: application for treatment of malignant disease. $J$ Virol. 2002;76:895-904.
33. Porosnicu M, Mian A, Barber GN. The oncolytic effect of recombinant vesicular stomatitis virus is enhanced by expression of the fusion cytosine deaminase/uracil phosphoribosyltransferase suicide gene. Cancer Res. 2003;63: 8366-8376.

34. Bateman A, Bullough F, Murphy S, et al. Fusogenic membrane glycoproteins as a novel class of genes for the local and immune-mediated control of tumor growth. Cancer Res. 2000;60:1492-1497.

35. Ebert O, Shinozaki K, Kournioti C, et al. Syncytia induction enhances the oncolytic potential of vesicular stomatitis virus in virotherapy for cancer. Cancer Res. 2004;64:3265-3270. 